Rev. Biol. Trop., 46(3):649-653, 1998

\title{
Composición bioquímica de la carne de Polymesoda radiata (Bivalvia: Corbiculidae) en Costa Rica
}

\author{
Eleazar Ruiz Campos ${ }^{1}$, Jorge Cabrera Peña², Rafael A. Cruz ${ }^{2}$ y José A. Palacios ${ }^{2}$ \\ Centro de Investigación en Ciencias del Mar (CIMAR), Universidad de Costa Rica, 2060 San José, Costa Rica. \\ Escuela de Ciencias Biologicas, Universidad Nacional, Heredia 86-3000, Costa Rica.
}

Recibido 23-IX-1997. Corregido 18-VI-1998. Aceptado 23-VI-1998.

\begin{abstract}
Monthly changes in biochemical composition and condition index of the green clam, Polymesoda radiata were studied from August 1992 through July 1993 in Bebedero river, Guanacaste, Costa Rica. This species has a continuous spawning throughout the year with maximun periods in December and April (following values are \% means and standard deviations). Fresh meat was $12.53 \pm 3.78$. Dry flesh compositions: $7829 \pm 2.15$ proteins; $9.52 \pm 1.00$ lipids; $5.78 \pm 2.50$ carbohydrates; and $6.43 \pm 2.10$ ashes. Water $90.10 \pm 2.14$ (correlated with the spawning period). The condition index was $9.89 \pm 2.14$. Dry flesh caloric value: $4.51 \pm 0.11 \mathrm{Kcal} / \mathrm{g}$. The condition index, caloric value and carbohydrates decreased during the spawning period. Lipids, proteins and ashes had not relationship with the spawning period. The mean condition index was inverse correlation with the spawning period.
\end{abstract}

Key words: Clam, bivalvia, Polymesoda radiata, condition index, energetic value, biochemical composition, Costa Rica.Beninger y Lucas (1984), Bressan y Marín (1985) y Páenz-Osuna et al. (1993), entre otros, han realizado estudios sobre la composición bioquímica de la carne de moluscos, debido a que éstos son importante en la dieta de los habitantes de las zonas costeras por la calidad de su carne y contenido de proteína.El cambio estacional en la composición bioquímica de los moluscos depende de parámetros del ambiente, como temperatura, salinidad, alimento disponible, productividad primaria y factores como la etapa del ciclo reproductivo en que se encuentren (Beninger y Lucas 1984, Ruiz et al. 1992). Lucas y Beninger (1985) realizaron un análisis teórico de los diferentes índices usados en acuacultura y determinaron que la relación entre peso de la carne seca y peso carne x 100, expresa la condición de los moluscos de una manera tan adecuada como otros índices que utilizan variables más difíciles de medir.

Los resultados de los estudios bioquímicos permiten determinar el valor alimenticio de la carne y proporcionan información que ayuda a entender el balance energético y ecológico de los moluscos (Giese y Pearse 1974). Por lo tanto se puede hablar de un ciclo bioquímico estrechamente relacionado con el ciclo gametogénico (Bayne 1976).En Costa Rica la composición químico-nutricional de moluscos no ha sido suficientemente estudiada y entre los trabajos efectuados a la fecha se encuentran los realizados por Báez (1985) en Polymesoda inflata; por Blanco y Montero (1992) en Strombus galeatus; por Cruz y Villalobos (1993) en Mytella guyanensis; y Jiménez-Arce (1993) en Strombus gracilior El objetivo de este trabajo fue determinar la composición química-nutricional de la carne e índice de condición de una población de Polymesoda radiata en el río Bebedero, Golfo de Nicoya, Costa Rica. 


\section{MATERIALES Y MÉTODOS}

Se trabajó un total de 978 ejemplares de $P$. radiata recolectados mensualmente al azar entre agosto de 1992 y julio de 1993 en un banco natural en la ribera izquierda del Río Bebedero a $5 \mathrm{Km}$ de Puerto Níspero, Costa Rica $\left(10^{\circ} 16^{\circ}\right.$ $44^{\prime \prime} \mathrm{N}$ y $85^{\circ} 14^{\prime} 37^{\prime \prime}$ O).Tras pesar, se removió y drenó la carne por media hora con papel absorbente; se pesó nuevamente y se deshidrató a $100^{\circ} \mathrm{C}$ por $24 \mathrm{hrs}$. para determinar el peso seco. Mensualmente se tomó una muestra de $10 \mathrm{~g}$ de carne seca, se molió y homogenizó para realizar los análisis bioquímicos proximales por triplicado. Se determinó el contenido de nitrógeno por el método de Kjeldhal (Anónimo 1980) y se convirtió a valores de proteína multiplicándolo por el factor 6.25 (Crips 1971). Los lípidos fueron obtenidos usando un extractor soxhlet con éter de petróleo (Anónimo 1980). Las cenizas fueron obtenidas por calcinación lenta, incrementado la temperatura hasta $500{ }^{\circ} \mathrm{C}$ donde se mantuvo por $5 \mathrm{hrs}$. Los carbohidratos fueron calculados por diferencia entre 100 menos el porcentaje de lípidos, proteínas y cenizas. Para calcular el valor del contenido calórico de la carne seca de $P$. radiata se utilizaron factores de conversión recomendados para moluscos: 5.7 $\mathrm{kcal} / \mathrm{g}$ para proteínas, $4.2 \mathrm{kcal} / \mathrm{g}$ para carbohidratos, $9.5 \mathrm{kcal} / \mathrm{g}$ para lípidos (Ansell et al. 1980, Beninger y Lucas 1984). Se realizó un
ANOVA entre el valor de los macroconstituyentes y los meses donde se encontró mayor porcentaje de organismos desovados, como también con los de menor desove. Se determinó el índice de condición (IC $=[$ Peso carne seca/peso carne fresca] x 100) según Lucas y Beninger (1985).

\section{RESULTADOS}

Las variaciones mensuales en los componentes bioquímicos de la carne de Polymesoda radiata (Cuadro 1), indican que los promedios fueron: humedad $90.1 \pm 2.14 \%$, cenizas $6.43 \pm$ $2.1 \%$, lípidos $9.52 \pm 1 \%$, proteínas $78.29 \pm$ $2.15 \%$ y carbohidratos $5.78 \pm 2.5 \%$. Las mayores fluctuaciones se producen en carbohidratos. El peso promedio de la carne fue de 12.52 $\pm 3.78 \%$. El porcentaje promedio de humedad de la carne de $P$. radiata presentó una correlación positiva $(\mathrm{r}=0.72 \mathrm{p}<0.05)$ con los meses de más alto porcentaje de organismos desovados (noviembre a abril) y con los meses donde el desove fue mínimo (mayo hasta octubre).Los carbohidratos no mostraron diferencias estadísticamente significativas $(p<0.05)$ entre las medias de los meses con desove y los de mínimo desove, sin embargo, existe una tendencia a su disminución en los meses de desove máximo. Las proteinas mostraron una relación inversa

\section{CUADRO 1}

Composición bioquímica y valor calórico $(V C)$ de la carne seca de $\mathrm{P}$. radiata, en la desembocadura del río Bebedero, Cañas, Guanacaste, Costa Rica (N mensual $=80 \pm 2$ ).

$\begin{array}{ccccccc}\text { Meses } & \text { Agua } & \text { Cenizas } & \text { Lípidos } & \text { Proteínas } & \text { Carbohidratos } & \text { VC } \\ & & & & & & \\ \text { Agosto (1992) } & 86.8 & 5.50 & 10.38 & 76.85 & 7.24 & 4.58 \\ \text { Setiembre } & 87.6 & 5.60 & 9.92 & 76.42 & 8.09 & 4.56 \\ \text { Octubre } & 87.8 & 4.50 & 8.90 & 76.46 & 10.19 & 4.55 \\ \text { Noviembre } & 91.5 & 5.60 & 12.12 & 78.78 & 3.48 & 4.68 \\ \text { Diciembre } & 91.6 & 8.50 & 9.17 & 77.32 & 5.01 & 4.40 \\ \text { Enero (1993) } & 92.4 & 6.20 & 8.80 & 79.30 & 5.72 & 4.48 \\ \text { Febrero } & 91.3 & 8.70 & 9.23 & 78.07 & 4.02 & 4.39 \\ \text { Marzo } & 92.2 & 7.60 & 9.36 & 80.93 & 2.08 & 4.45 \\ \text { Abril } & 91.9 & 5.20 & 8.39 & 80.76 & 5.63 & 4.50 \\ \text { Mayo } & 89.8 & 4.90 & 9.51 & 82.29 & 3.35 & 4.57 \\ \text { Junio } & 87.3 & 10.90 & 8.70 & 75.18 & 5.23 & 4.30 \\ \text { Julio } & 91.3 & 4.00 & 9.81 & 77.15 & 9.32 & 4.63\end{array}$


con los carbohidratos $(r=-0.62, p<0.05)$. Las proteína, lípidos y ceniza no presentan estadísticamente diferencias significativa entre los promedios de las medias, lo que indica que no hay relación específica alguna entre estos tres parámetros bioquímicos con los meses de desove y con los de mínimo desove. El valor calórico $(\mathrm{kcal} / \mathrm{g})$ presentó diferencias significativas entre las medias de los meses en desove con un promedio de $4.44 \pm 0.05 \%$ y los meses de desove mínimo con un promedio de $4.55 \pm 0.12 \%$ $(\mathrm{p}<0.05)$.Los valores mensuales del índice de condición y su desviación estándar (Fig. 1) indican que los más altos se obtuvieron entre agosto (13.20\%) y octubre (12.17\%), así como entre mayo $(10.18 \%)$ y julio $(8.70 \%)$, lo que coincide con la época lluviosa en la zona de estudio (mayo hasta octubre). Se obtuvo una correlación inversa entre el porcentaje de individuos desovados y los valores promedio del índice de condición ( $\mathrm{r}=-0.72 ; \mathrm{p}<0.05)$. El promedio anual del índice de condición para $P$. radiata fue de $9.89 \pm 2.14 \%$ con un coeficiente de variación de 22.58 .

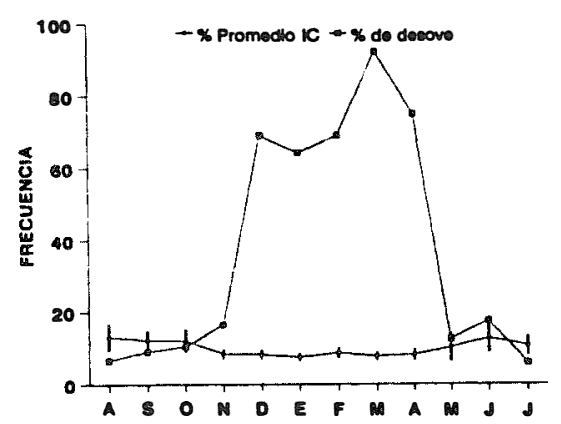

Fig. 1. Frecuencia del índice de condición promedio \pm desviación estándar y porcentaje de individuos desovados, obtenidos mensualmente para Polymesoda radiata en el río Bebedero, Guanacaste, Costa Rica.

\section{DISCUSION}

El peso promedio de carne fresca en $P$. radiata $(12.53 \pm 3.78 \%)$, es inferior al reportado para Protothaca asperrima (22.0\%) por Palacios (1985) en Colorado de Abangares, Guanacaste,
Costa Rica; para Crassostrea rizhophorae (18.17 \%) por Cabrera et al. (1983) en Limón, Costa Rica; para Anadara tuberculosa (16.31 y $20.34 \%$ ) por Cruz y Palacios (1983) en Punta Morales, Costa Rica y para Modiolus capax (24.60-27.20\%) por Cabrera et al. (1995) en Playa Ocotal, Costa Rica. Semejante al obtenido para Polymesoda inflata (10.87 \%) por Báez (1985) en el manglar de Pochote, Puntarenas, Costa Rica y para Pinctada mazatlanica (13.89 \%) por Solano et al. (1997) en Golfo de Nicoya, Costa Rica y mayor que el informado para Crassostrea columbiensis $(9.1 \pm 4.11 \%)$ por Caballero et al. (1996-1997) en Punta Morales, Costa Rica. La correlación positiva encontrada entre la humedad de la carne y los meses con mayor y menor desove coincide con lo informado para Donax vittatus por Ansell (1972) en Kames Bay; para Polymesoda inflata por Báez (1985) en el Manglar de Pochote, Costa Rica y para Mytella guayanensis por Cruz y Villalobos (1993) en Golfo de Nicoya, Costa Rica. La tendencia de los carbohidratos a disminuir durante los meses de mayor desove, coincide con lo informado para Crassostrea iridescens y C. corteziensis por Páenz-Osuna et al. (1993) en las costa Oeste de México. La relación inversa encontrada entre las proteína y los carbohidratos, coincide con lo reportado para Mytilus galloprovincialis por Bressean y Marín (1985) en laguna de Venecia, Mar Adriático y para Crassostrea iridescens y C. corteziensis por Páenz-Osuna et al. (1993) en las costa Oeste de México.La correlación inversa obtenida entre el porcentaje de individuos desovados y los valores promedio del índice de condición, coincide con lo informado para bivalvos marinos por Lucas y Beninger (1985), para Anadara granosa por Broom (1983) y para Ostrea iridescens por Fournier (1992) en la costa pacífica de Costa Rica.El promedio anual del índice de condición en $P$. radiata $(9.89 \pm 2.14 \%)$, difiere con lo informado para Anadara granosa (16.0 a $18 \%)$ por Broom (1983); para Ostrea iridescens (14.0 a $29.0 \%$ ) por Fournier (1992); para Pinctada mazatlanica $(18.92 \pm 4.40)$ por Solano et al. (1997) en Golfo de Nicoya, Costa Rica; y para Crassostrea columbiensis $(11.79 \pm 5.02 \%)$ por 
Caballero et al. (1996-1997)en Punta Morales, Costa Rica.La relación existente entre las fluctuaciones mensuales del valor del índice de condición y el ciclo reproductivo en $P$. radiata coinciden con lo informado por Bressam y Marín (1985) y Fernández y De Vittio (1987).Lo anteriormente descrito permite inferir que $P$. $r a$ diata se comporta como una especie típica de las zonas tropicales, que el índice de condición es un buen indicador del ciclo reproductivo de la especie y que el contenido de proteína demuestra el valor nutritivo de ésta.

\section{RESUMEN}

Se determinó la composición bioquímica y el indice de condición de la almeja verde (Polymesoda radiata) en el río Bebedero, Guanacaste, Costa Rica, entre agosto de 1992 y julio de 1993. El peso promedio de carne fresca fue de $12.53 \pm 3.78 \%$, con un valor promedio de humedad de $90.10 \pm 2.14 \%$. El promedio de los componentes en base seca fue proteínas $78.29 \pm 2.15$, lípidos $9.52 \pm 1 \%$, carbohidratos $5.78 \pm 2.5 \%$ y cenizas $6.43 \pm$ $2.1 \%$. El promedio del índice de condición para la población fue de $9.89 \pm 2.14$. El promedio del valor calórico fue $4.51 \pm 0.11 \mathrm{Kcal} / \mathrm{g}$. Se determinó que el índice de condición, valor calórico y carbohidratos decrecen con la época reproductiva. Los lípidos, proteínas y cenizas no tiene relación con el ciclo reproductivo.

\section{REFERENCIAS}

Anónimo. 1980. Official methods of Analysis of the AOAC. Washington, D. C. $1141 \mathrm{p}$.

Ansell, A. 1972. Distribution, growth and seasonal changes in biochemical composition for the bivalve Donax vittatus (da Costa) from Kames Bay, Millport. J. Exp. Mar. Biol. Ecol. 10:137-150.

Ansell, A., L. Frenkiel \& M. Moueza. 1980. Seasonal changes in tissue weight and biochemical composition for the bivalve Donax trunculus L. On the Algerian coast. J. Exp. Mar. Biol. Ecol. 45:105-116.

Báez, R. 1985. Evaluación de algunos aspectos de la biología del molusco Polymesoda inflata (Philipp, 1851) (Bivalvia: Corbiculidae), en el manglar de Pochote, Puntarenas, Costa Rica. Tesis de Licenciatura. Escuela de Biología, Universidad de Costa Rica, San José, Costa Rica. 70 p.
Bayne, B. 1976. Aspects of reproduction in bivalve molluscs. In Estuarine processes. Vol. 1. Academic, Londres. $541 \mathrm{p}$.

Beninger, P. \& A. Lucas. 1984. Seasonal variation in condition, reproductive activity and gross biochemical composition of two species of adult clam reared in a common habitat Tapes decussatus (L) (Jefreys) and Tapes philippinarum (Adams and Reeve). J. Exp. Mar. Biol. Ecol. 79:19-37.

Blanco, A. \& M. Montero. 1992. Composición químico-nutricional de la carne de cambute, Strombus galeatus (Mesogastropoda:Strombidae). Rev. Biol. Trop. 40:89-93.

Bressan, M. \& M. Marín. 1985. Seasonal variations in biochemical composition and condition index of cultured mussels (Mytilus galloprovincialis (LMK)) in the lagoon of Venice (North Adriatic). Aquaculture 48:13-21.

Broom, M. 1983. Gonad development and spawning in Anadara granosa (L) (Bivalvia: Arcidae). Aquaculture 30: 211-219.

Caballero, A. F., J. Cabrera \& Y. Solano. 1996-1997. Descripción del crecimiento y madurezsexual de una población de Crassostrea columbiensis (Mollusca: Bivalvia). Rev. Biol. Trop. 44-45:335-339.

Cabrera, J., E. Zamora \& O. Pacheco.1983. Determinación del tamaño comercial de la ostra de manglar Crassostrea rizhophorae (Guilding 1828) en sistema de cultivo suspendido en estero Vizcaya, Limón, Costa Rica. Rev. Biol. Trop. 31:257-261.

Cabrera, J., R. A. Cruz , Y. Solano \& M. Protti. 1995. Biometría de Modiolus capax (Bivalvia: Mytilidae) en Playa Ocotal, Guanacaste, Costa Rica. Rev. Biol. Trop. 43:173-176.

Crisp, J. 1971. Energy flow measurement In N. A. Holmey A.D. Mc Intypre. Methods for the study of marine benthos. IBP Handbook $N^{\circ} 16$. Blackwell, Oxford. 249 p.

Cruz, R. A. \& J. A. y Palacios. 1983. Biometría del molusco Anadara tuberculosa (Pelecypoda: Arcidae) de Punta Morales, Puntarenas, Costa Rica. Rev. Biol. Trop. 31:45-50.

Cruz, R. A. \& C. Villalobos 1993. Monthly changes in tissue weight and biochemical composition of the mussel Mytella guyanensis (Bivalvia: Mytillidae) in Costa Rica. Rev. Biol. Trop. 41:93-96.

Fernández, N. \& N. de Vitto. 1987. Composición bioquímica, índice de condición y valor energético de la Ostrea puelchana (B'orbigny), relacionándolas con el ciclo reproductivo. J. Exp. Mar. Biol.Ecol. 108:113-12. 
Fournier, M. 1992. The reproductive biology of the tropical rocky oyster Ostrea iridescens (Bivalvia: Ostreidae) on the Pacific coast of Costa Rica. Aquaculture 101:371-378.

Giese, A. \& J. Pearse. 1974. Introduction general principles. In. Reproduction of marine invertebrates. A. C. Giese y J. S. Pearse, (eds). Vol. I. Academic, Nueva York. 546 p.

Jiménez-Arce, G. 1993. Composición químico-nutricional en diferentes tallas y sexos del cambute, Strombus gracilior (Mesogastropoda:Strombidae) de Playa Panamá, Costa Rica. Rev. Biol. Trop. 41:345-349.

Lucas, A. \& P. Beninger. 1985. The use of physiological condition indices in marine bivalvia aquaculture. Aquaculture 44:187-200.

Páenz-Osuna, F., H. Zozueta-Padilla \& J. Osuna-López. 1993. Biochemical composition of the oysters Crassostrea iridescens (Hanley) and Crassostrea corteziensis (Hertlein) in the north-west coast of
México: Seasonal changes. J. Exp. Mar. Biol. Ecol. 170:1-9.

Palacios, J. 1985. Biometría, crecimiento y maduración sexual en la almeja blanca Protothaca asperrima (Pelecypoda: Veneridae) en Colorado de Abangares, Guanacaste, Costa Rica. Tesis de Licenciatura. Escuela de Ciencias Biológicas, Universidad Nacional, Heredia, Costa Rica. 85 p.

Ruiz, C. D. Martínez, G. Mosquera, M. Abad \& J. Sánchez. 1992. Seasonal variation in condition, reproductive activity and biochemical composition of the flat oyster, Ostrea edulis, from San Cibran (Galicia, Spain). Mar. Biol. 112:67-74.

Solano, Y. J. Cabrera, J. A. Palacios \& R. A. Cruz. 1997. Madurez sexual, índice de condición y rendimiento de Pinctada mazatlanica en Isla Pan de Azúcar, Golfo de Nicoya, Puntarenas, Costa Rica. Rev. Biol. Trop. 45:1049-1054. 